\title{
Innovación en la enseñanza de la Interacción Persona-Ordenador: interfaces imaginadas, ciencia-ficción y trabajo con usuarios reales Innovation in teaching Human-Computer Interaction: imagined interfaces, sci-fi and working with real users
}

\author{
Roberto Therón ${ }^{1,2}$, Juan Cruz-Benito ${ }^{1,2}$, Felicidad García-Sánchez ${ }^{1,3}$, Rodrigo Santamaría², Francisco J. García- \\ Peñalvo ${ }^{1,2}$ \\ \{theron, juancb, felicidadgsanchez, rodri, fgarcia\}@usal.es \\ ${ }^{1}$ Grupo de Investigación GRIAL \\ Universidad de Salamanca \\ Salamanca, España \\ ${ }^{3}$ Departamento de Historia \\ del Arte-Bellas Artes \\ Universidad de Salamanca \\ Salamanca, España
}

\section{INTRODUCCIÓN}

Resumen- La idea de las interfaces imaginadas y el uso de los recursos y contenidos recogidos en obras de ciencia ficción no es nuevo en la búsqueda de motivación, temáticas e inspiración en el mundo de la informática. La historia del cine y la televisión, en multitud de obras que se podrían adscribir al género de la CienciaFicción, han mostrado a los espectadores imaginativas formas de satisfacer necesidades humanas a través de la intervención de poderosas máquinas dotadas de inteligencia artificial que se comunicaban con las personas de muy diversas formas. En este artículo se presenta una experiencia de innovación docente para la asignatura Interacción Persona-Ordenador del Grado en Ingeniería Informática. En esta experiencia se utiliza este concepto de interfaces imaginadas y el trabajo con usuarios reales como base para incrementar la motivación y el aprendizaje de la asignatura por parte de los estudiantes. Estos conceptos y formas de trabajo se aplican a través de la adaptación de los trabajos prácticos a sus preferencias temáticas y contexto personal, o trabajando con usuarios reales en todas aquellas prácticas que lo permitan, de modo que se pueda llegar a un contexto práctico quasi-real que mejore el aprendizaje y los resultados.

\section{Palabras clave: Interacción Persona-Ordenador, IPO, Innovación docente, ciencia-ficción, usuarios reales}

Abstract- The idea of imagined interfaces and the use of resources and content related to sci-fi is not new in the search for motivation, topics or inspiration in computer sciences. In the history of cinema and TV, a lot of works that could be ascribed to the science-fiction genre, have shown to the spectator imaginative ways of satisfying human needs through the intervention of powerful machines equipped with artificial intelligence that communicate with the people in many ways. This paper presents an experience of teaching innovation for the Human-Computer Interaction subject within the Degree in Computer Engineering. This experience uses this concept of imagined interfaces and work with real users as a basis to increase students' motivation and learning results through the adaptation of practical work to their topic preferences and personal context; as well as through working with real users in all practical assignments proposed in the subject, in order to provide to the students a quasireal practical context that could improves learning and its results.

Keywords: Human Computer-Interaction, HCI, Teaching innovation, sci-fi, imagined interfaces, real users
En este artículo se comenta una experiencia de innovación docente llevada a cabo en el segundo cuatrimestre del curso 2016-2017, concretamente en el ámbito de la asignatura Interacción Persona-Ordenador del tercer curso del Grado en Ingeniería Informática.

A modo de resumen, dentro de la innovación docente aplicada y comentada en este artículo, se pretende proporcionar temáticas interesantes y motivadoras para los trabajos prácticos de la asignatura (a través de la ciencia ficción y las interfaces imaginadas), adaptar parte de la asignatura a los gustos de los estudiantes permitiendo que elijan en parte la temática de sus prácticas, promoción del trabajo en condiciones quasi-reales por parte de los estudiantes en las prácticas que involucren pruebas o entrevistas con usuarios, la realización de actividades complementarias a la asignatura que amplíen la visión de los estudiantes sobre cuestiones propias o relacionadas con la asignatura, o la sustitución de los trabajos habituales de fin de asignatura para realizar prácticas colaborativas entre estudiantes que conlleven ir un paso más allá (saltando de supuestos teórico-prácticos a experiencias mucho más completas y prácticas) para los alumnos que lo elijan.

Para ello, se apuesta por el trabajo con el concepto de interfaces imaginadas (aquellos puntos de conexión humanomáquina no existentes actualmente), la ciencia ficción o el trabajo con usuarios reales, de acuerdo con una corriente internacional de autores e investigadores que proponen trabajar en este ámbito como modo de innovación en la Interacción Persona-Ordenador, tanto en el ámbito profesional (Jordan, Mubin, \& Silva, 2016; Marcus, 2013, 2014, 2015; Schmitz, Endres, \& Butz, 2008; Sterling, 2013) como en el docente (Golbeck, 2017).

Buena parte de las actividades, fundamentación, resultados $\mathrm{y}$ propuestas se encuentran en la web creada por los responsables del proyecto de innovación docente http://vis.usal.es/ interfacesimaginadas/. 
En las siguientes secciones se proporciona una visión más detallada del proyecto y sus fundamentos (sección 2 Contexto), sus aportaciones y acciones (sección 3, Descripción), los resultados conseguidos (sección 4, Resultados) o una discusión sobre aquello conseguido y cómo esta experiencia se puede aplicar en distintos cursos o asignatura (sección 5, conclusiones).

\section{CONTEXTO}

¿Qué es la Interacción Persona-Ordenador como disciplina? De acuerdo con el Special Interest Group in Computer Human Interaction de la Asociación ACM (Hewett et al., 1992) y (Abascal et al., 2001): "Es la disciplina relacionada con el diseño, evaluación y implementación de sistemas informáticos interactivos para el uso de seres humanos, y con el estudio de los fenómenos más importantes con los que está relacionado”.

Por otro lado, la Interacción Persona-Ordenador, como asignatura, es una disciplina introducida en el currículum de los nuevos planes de estudio del Grado en Ingeniería Informática implantados a partir de la adaptación de las Ingenierías en Informática al contexto del marco de Bolonia. Que esta asignatura sea de reciente creación, tiene diversas implicaciones: es una asignatura aún en evolución y en fase de afianzarse, falta de vicios y tradiciones dentro del contexto de las ingenierías. Esto hace que sea una asignatura donde los profesores pueden usar la innovación como forma de experimentar la mejora de la enseñanza de una disciplina novedosa dentro de los planes de estudios; una asignatura que presenta una serie de contenidos cercanos a las últimas tendencias en la ingeniería informática, y por tanto, no solo es que se pueda aprovechar de la innovación, sino que la necesita como vehículo transmisor de conocimiento y elemento motivador en un contexto tan cambiante como la informática y tan aplicable por parte de los alumnos en el futuro profesional que les espera nada más finalizar sus estudios (Michavila, Martínez, Martín-González, García-Peñalvo, \& Cruz-Benito, 2016).

Como se ha comentado en la sección de introducción, la experiencia de innovación docente presentada en este artículo se basa en dos puntos principales: la aplicación de temáticas que puedan motivar a los estudiantes (como la ciencia ficción o las interfaces imaginadas) y el trabajo aplicado cercano a la experiencia real (trabajando con usuarios reales dentro de la asignatura). Estos principios se comentan a continuación.

La idea de las interfaces imaginadas y el uso de los recursos y contenidos recogidos en obras de ciencia ficción no es nuevo en la búsqueda de motivación, temáticas e inspiración en el mundo de la informática. La historia del cine y la televisión, en multitud de obras que se podrían adscribir al género de la Ciencia-Ficción han mostrado a los espectadores imaginativas formas de satisfacer necesidades humanas a través de la intervención de poderosas máquinas dotadas de inteligencia artificial que se comunicaban con las personas de muy diversas formas. Esta representación de interfaces de usuario imaginadas se ha exacerbado durante el siglo XXI —el ejemplo paradigmático es la interfaz gestual de la película Minority Report (Steven Spielberg, 2002) - y ha tenido un fuerte impacto en la sociedad actual, muchas veces incorporando a la vida real réplicas casi exactas de lo que no hace mucho eran interfaces de ciencia ficción - de nuevo, el ejemplo de Minority Report, con la aparición del controlador gestual de juegos Kinect en 2009, es el caso más representativo. Sin embargo, lo que es menos conocido, en realidad lo que el director Steven Spielberg mostraba al gran público en su película era una propuesta de interfaz de usuario que John Underkoffler venía investigando desde el siglo pasado en el MIT (Underkoffler, Parent, \& Kramer, 2009). Esta simbiosis observada entre la ciencia-ficción y el mundo académico o profesional relacionado con la informática prueba el buen funcionamiento del binomio, y hace que tenga sentido utilizar los conceptos de interfaz imaginada como método de innovación y motivación en el contexto de una asignatura académica como la Interacción Persona-Ordenador. Además, este binomio no está siendo únicamente propuesto por los docentes y colaboradores en esta asignatura, sino que se está empezando a plantear en otras universidades y titulaciones relacionadas con la informática a lo largo del mundo (Golbeck, 2017).

Por otra parte, en la asignatura y su relación con el contexto actual en el que se desenvuelve la informática, se percibe esencial que los alumnos consigan cierta experiencia práctica dentro de su aprendizaje. Dentro de la asignatura, durante los años que se ha impartido se ha hecho hincapié en aplicar los conceptos teóricos de forma práctica, a través de trabajos entregados por los alumnos. Estos trabajos relacionados con la Interacción Persona-Ordenador cubren algunos aspectos que incluyen a usuarios directamente, como pueden ser aquellos relacionados con la búsqueda de necesidades de los usuarios, la evaluación de prototipos o la evaluación de sistemas y aplicaciones ya implementados. Es por ello que, como parte de la innovación docente llevada a cabo, se ha hecho especial hincapié en que estas prácticas se hayan llevado a cabo con usuarios reales (en muchos casos otros compañeros, familiares, compañeros de facultad, o simples viandantes que caminan al lado de la facultad en el caso de las prácticas de búsqueda de necesidades de usuarios).

Como objetivos concretos de la propuesta de innovación docente, se pueden plantear los siguientes:

- $\quad$ Propuesta de temáticas interesantes y motivadoras para el estudiante dentro de los trabajos prácticos. Especialmente centrándose en la ciencia ficción, a sabiendas que hay un gran número de estudiantes de informática aficionados a ella.

- Adaptación a los gustos e intereses de los estudiantes a través de la elección de éstos de los temas concretos dentro de la línea de interfaces imaginadas y ciencia ficción para sus trabajos prácticos.

- Adecuación de las prácticas a contextos quasireales de experimentación con usuarios, a través de la interacción con usuarios fuera del entorno habitual de la clase o la asignatura.

- Realización de actividades complementarias a la asignatura para aquellos alumnos que lo deseen. Siguiendo la línea de las interfaces imaginadas y la ciencia ficción, la propuesta se basó especialmente en sesiones de cinefórum.

- Adaptación a los distintos contextos, tipos de aprendizaje y niveles de los alumnos, permitiendo sustituir el trabajo final de la asignatura por otra propuesta en la que se realiza un trabajo más 
ambicioso de forma colaborativa por un grupo de estudiantes voluntarios.

El proyecto de innovación docente que se describe se enclava en la asignatura Interacción Persona-Ordenador, asignatura troncal del tercer curso del Grado en Ingeniería Informática impartida en la Universidad de Salamanca. El público objetivo son sus alumnos (115 matriculados), hombres y mujeres, los cuales tienen típicamente en el caso de este estudio entre 20-29 años.

\section{DESCRIPCIÓN}

Como se ha apuntado previamente en la descripción de los objetivos, para conseguirlos se han realizado distintas aproximaciones y actividades en la asignatura para hacerla más dinámica, más interesante para los alumnos o con más posibilidades de adaptación a los intereses de los estudiantes.

Los distintos vectores de innovación aplicados durante la experiencia de innovación en la asignatura han sido:

1. Temática de trabajos: como línea general dentro de la asignatura, se ha seguido la idea de las interfaces imaginadas dentro de los distintos trabajos realizados dentro de la evaluación continua o del trabajo final. Los trabajos en sí han seguido el guión de otros cursos, pero se ha incorporado el punto de vista de las interfaces imaginadas o la ciencia ficción para motivar a los estudiantes, estimular su creatividad o para crear experiencias de aprendizaje únicas en las que todos los trabajos se rijan por las mismas reglas, pero puedan resultar distintos en función del tema imaginado que plantee el estudiante.

2. Actividades con requisitos de trabajo con usuarios. En la Interacción Persona-Ordenador, como se puede imaginar, el usuario es una parte fundamental; es el usuario en el que se centra todo el trabajo, valida las soluciones, proporciona la información básica sobre la que desarrollar el trabajo, etc. Durante la asignatura se desarrollan diversos trabajos en los que se busca que el usuario proporcione información sobre sus gustos, necesidades, opinión sobre los sistemas ya existentes o que puedan diseñar los alumnos, etc. Durante este curso, se ha hecho especial hincapié en que el trabajo con usuarios salga del ámbito habitual de la clase/asignatura, intentando llegar a lo que podríamos denominar usuarios reales. Se entiende que estos usuarios reales son personas fuera del ámbito de la asignatura, y que pueden proporcionar puntos de vista no sesgados, no orientados, o no influidos por participar en el proceso de trabajo relacionado con la temática de la asignatura.

3. Sesiones complementarias de cinefórum. Además de la actividad habitual de la clase, este año se han incluido distintas sesiones de visionado de películas fuera del horario lectivo. Estas películas seleccionadas tenían un alto grado de innovación o eran notables por su impacto en el cine respecto a las interfaces imaginadas que presentan. Durante estas sesiones, los responsables del proyecto de innovación han ido preparando distintas partes de las películas sobre las cuáles exponer un tema concreto que afecte a la disciplina en la actualidad o que pueda suscitar debate a cualquier nivel (no solo tecnológico, sino ético, de innovación, etc.), de modo que los estudiantes asistentes puedan discutir distintos aspectos relacionados que amplían lo que se ve durante las horas lectivas o lo hagan desde un prisma distinto al de la clase habitual.

4. Análisis de películas. Como trabajo complementario a los propuestos habitualmente en la asignatura, y de modo que pueda servir como motivación y comprensión para el resto de las prácticas y la temática subyacente de interfaces imaginadas, se ha propuesto un trabajo extra donde cada uno de los alumnos ha elegido una película de un listado propuesto por los profesores, entregando un informe que en el que se analiza el film desde la perspectiva de la asignatura y la Interacción Persona-Ordenador.

5. Grupo especial de trabajo. Para plantear una experiencia más completa y trabajar desde otro punto de vista con los alumnos más interesados en la asignatura (y en las interfaces imaginadas), se ha establecido un grupo especial de trabajo formado por estudiantes voluntarios. A este grupo de trabajo, se le ha propuesto la realización de un trabajo fin de asignatura distinto al de sus compañeros, de modo que, en vez de proponer una interfaz imaginada de forma teórica (siguiendo el proceso de diseño centrado en el usuario (Norman, 1988, 2013; Norman \& Draper, 1986) como todos los compañeros), puedan plantearla de forma teórica para después implementarla en la medida de las posibilidades técnicas disponibles.

\section{Resultados}

Como resultados tangibles de la innovación docente en la asignatura, se pueden destacar los siguientes (relacionados con las propuestas:

1. De los seis trabajos prácticos propuestos dentro de la evaluación continua (30\% de la nota de la asignatura), cuatro de ellos estaban fundamentados en el trabajo con interfaces imaginadas. Del mismo modo, el trabajo final de la asignatura (30\% de la nota final) ha estado orientado totalmente al trabajo con interfaces imaginadas.

2. En todos los trabajos que implican la participación de usuarios (3 de evaluación continua y el trabajo final), los alumnos han trabajado de forma habitual con usuarios reales fuera de sus compañeros de clase. Esto se ha valorado de forma positiva en la evaluación numérica (nota) de los trabajos, lo que les ha animado. A continuación, cuando se muestren más resultados se verá la opinión de los estudiantes sobre este trabajo con usuarios reales.

3. Se han hecho cuatro sesiones de cinefórum complementarias a la asignatura a lo largo del trimestre que duran las clases. Las películas y 
series elegidas para ello han sido: "Regreso al futuro 2", "2001: una odisea del espacio", "Westworld” y "Metrópolis". Estas sesiones de cine han tenido una tibia acogida y una asistencia excesivamente variable. Típicamente, a ellas han asistido entre 5 y 30 estudiantes (figura 1).

4. Como trabajo dentro de la evaluación continua de la asignatura se propuso que cada uno de los estudiantes hiciese un análisis crítico desde el punto de vista de la Interacción PersonaOrdenador de una película a su elección (de un listado propuesto por los responsables de la asignatura). Además de contar como trabajo, se planteó a los alumnos que las fichas de análisis crítico serían públicas en la web creada para informar del proyecto. Se han realizado 122 fichas (entre profesores y alumnos). El listado completo de fichas de análisis realizadas está disponible en http://vis.usal.es/ interfacesimaginadas/index.php? id $=108$

5. Se ha formado un grupo de trabajo, compuesto por 10 estudiantes voluntarios. Estos han trabajado durante dos meses en el trabajo, grupal y colaborativo, propuesto como alternativa a la práctica individual final de la asignatura. Como se ha comentado, en este trabajo no solo se pedía una aproximación teórica a la materia, sino llegar a una implementación real mínima de la interfaz imaginada. En el momento en el que se escribe este artículo, esta interfaz imaginada está siendo acabada y no ha sido evaluada por los profesores.

Para evaluar el impacto del proyecto y de los distintos vectores de innovación introducidos dentro de la asignatura se han planteado dos métodos: una encuesta de satisfacción focalizada sobre los aspectos del proyecto de innovación (e independiente de la encuesta que realiza la Universidad para recabar datos sobre la calidad de la enseñanza) y una comparativa de notas entre los distintos grupos de estudiantes que la han cursado este año (aquellos que han participado en el grupo especial de trabajo frente a los que no) y entre los estudiantes de este año y los de años anteriores.

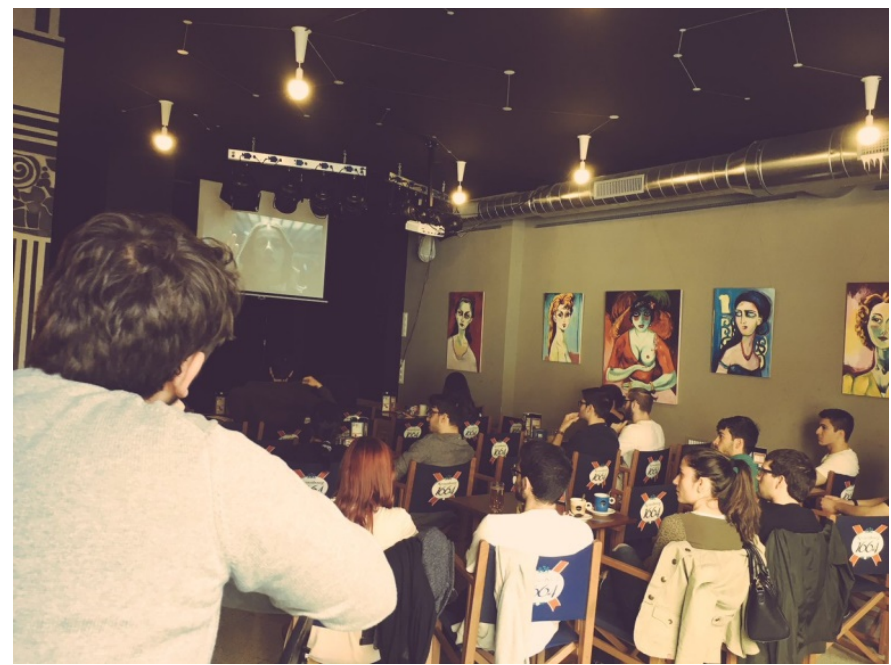

Figura 1. Sesión de cinefórum sobre la serie "Westworld" https://twitter.com/felizgsanchez/status/855452040440492034
Sobre el primer método de evaluación se comentan algunas evidencias recabadas en el momento de la escritura de este artículo, ya que el cuestionario utilizado como evaluación aún está siendo utilizado: el plazo para que los estudiantes hagan su valoración cerrará después del fin de la segunda convocatoria de la asignatura, en Julio de 2017. Sobre el segundo método de evaluación no se exponen datos o conclusiones ya que la evaluación no ha acabado (la asignatura se cierra a principio de Julio de 2017). En el caso de este segundo método de evaluación, los responsables evidencian una limitación, y es que los resultados pueden no ser comparables al $100 \%$ con otras ediciones de la asignatura ya que ha participado un profesor nuevo en esta convocatoria.

Respecto al cuestionario enviado a los estudiantes, este se ha implementado utilizando Google Forms y se ha distribuido en los foros de la plataforma Moodle que da soporte a la asignatura. De los 115 alumnos matriculados, en el momento que se escribe este artículo han participado $32-27,82 \%$ de los estudiantes- (el plazo para participar se cierra en Julio de 2017). A continuación, se comentan las preguntas del cuestionario y algunos de los resultados más relevantes:

Las preguntas del cuestionario han sido las siguientes:

1. Sexo (hombre/mujer/prefiero no decirlo)

2. Edad (número)

3. ¿Es la primera vez que cursas la asignatura? (Sí/no)

4. ¿Cuál es tu satisfacción en general con la asignatura? (Escala Likert con valores 1-5, 1 nada satisfecho, 5 muy satisfecho).

5. $\quad \dot{¿}$ Te parece acertado usar las interfaces imaginadas / ciencia ficción como hilo conductor de la asignatura? (Escala Likert con valores 1-5, 1 nada acertado, 5 totalmente acertado).

6. ¿Te ha motivado la temática a la hora de enfrentarte a los trabajos de la asignatura? (Escala Likert con valores 1-5, 1 ninguna motivación, 5 mucha motivación).

7. ¿Has asistido a alguna sesión de cinefórum propuesta como complemento a la asignatura? (Sí/no)

8. ¿A qué películas has asistido dentro de las sesiones de cine? (Se pueden seleccionar los títulos de las películas/series proyectadas)

9. ¿Has participado en el grupo especial de trabajo sobre Interfaces Imaginadas? (Sí/no)

10. Si has participado en ese grupo... ¿Cuál es tu satisfacción general con la propuesta de trabajo? (Escala Likert con valores 1-5, 1 nada satisfecho, 5 muy satisfecho).

11. Si has participado en ese grupo... ¿Crees que te ha beneficiado a la hora de aprender más en la asignatura? (Escala Likert con valores 1-5, 1 nada beneficionso, 5 muy beneficioso).

12. ¿Qué te parece haber trabajado con usuarios reales en las prácticas? (Escala Likert con valores 1-5, 1 muy mal, 5 muy bien). 
13. Señala algo que mejorarías de la asignatura (respuesta abierta).

14. Señala algo que te haya gustado de la asignatura (respuesta abierta).

Sobre los resultados del cuestionario, se van a comentar a continuación de forma breve los más relevantes. De los 32 estudiantes que han participado en el cuestionario, el 71,9\% son hombres, el $21,9 \%$ son mujeres y otro $6,3 \%$ prefiere no revelar su sexo, todos ellos tienen una edad comprendida entre 20 y 29 años, siendo la media de edad de 22 años. De los participantes, como métrica extra, se sabe que el 6,3\% han cursado la asignatura previamente, mientras que el 93,7\% la están cursando por primera vez.

\section{¿Cuál es tu satisfacción en general con la asignatura?} 32 responses

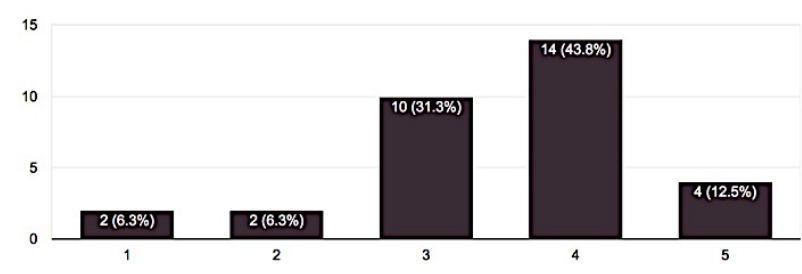

Figura 2. Distribución de valoraciones de los estudiantes sobre su satisfacción general con la asignatura.

¿Qué te parece haber trabajado con usuarios reales en las prácticas? 32 responses

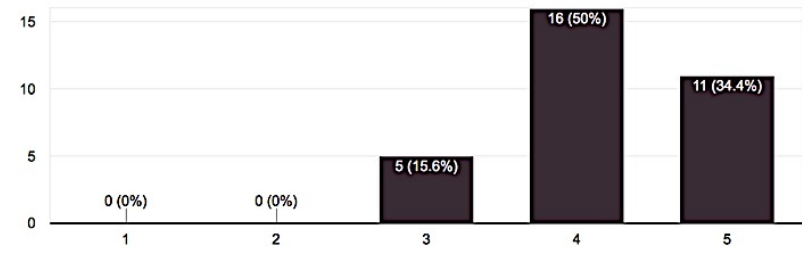

Figura 3. Distribución de valoraciones de los estudiantes de su opinión sobre haber trabajado con usuarios reales en las prácticas.

Acerca de su opinión sobre los distintos aspectos consultados, se obtiene que los estudiantes tienen una opinión general sobre la asignatura con una puntuación de 3,5/5 (figura 2). Acerca de la opinión sobre si les ha parecido acertado usar la temática de las interfaces imaginadas, en general parece que los estudiantes aprueban la propuesta, otorgándole de media una puntuación de 3,47 / 5. Del mismo modo, parece que esta propuesta temática les motiva a la hora de enfrentarse a la asignatura, ya que le dan una puntuación en la escala Likert de $3,53 / 5$.

Sobre la asistencia a las distintas sesiones de cinefórum, solo el $21,9 \%$ de los estudiantes dicen haber asistido a alguna de ellas.

De los 32 participantes actuales en la encuesta, el 12,5\% (4 estudiantes de los 10 participantes en total) declaran haber participado en el grupo especial de trabajo sobre interfaces imaginadas. Su satisfacción general con la propuesta de trabajo que se les planteó es bastante buena, ya que se obtiene una puntuación de 4,25/5 puntos en la escala Likert. Del mismo modo, estos participantes creen en general que participar en ese grupo especial les ha beneficiado a la hora de aprender más en la asignatura (puntuación de 4,5/5).

Por último, a la hora de valorar la experiencia de trabajo con usuarios reales, los estudiantes están de acuerdo en señalar que ha esta experiencia ha sido bastante positiva (puntuación de 4,19/5 en la escala Likert, figura 3).

\section{CONCLUSIONES}

En general, se puede concluir que la propuesta de innovación docente aplicada en este curso ha sido bastante positiva. De forma inicial, la propuesta de innovación basada en las interfaces imaginadas, ciencia ficción y trabajo con usuarios parecía adecuada a la hora de motivar a los estudiantes, adaptar los trabajos a sus gustos, o incluso adaptar parte de la asignatura a los intereses de aprendizaje de parte de los estudiantes; además de proporcionar un entorno de aprendizaje práctico mejorado sobre los años previos de la asignatura.

De acuerdo con la valoración de los estudiantes y las evidencias recogidas mediante las encuestas (y por lo percibido en entrevistas personales con los estudiantes), se puede afirmar que los estudiantes tienen una buena opinión sobre las distintas innovaciones introducidas en la asignatura de acuerdo a sus opiniones.

Del mismo modo, como se ha comentado previamente, falta una valoración más completa comparando las notas finales obtenidas por los estudiantes este año frente a los estudiantes de otros años, o comparando las notas de los estudiantes que han participado en el trabajo del grupo especial sobre interfaces imaginadas, de modo que se pueda vislumbrar si el trabajo con estas innovaciones ha mejorado o no el aprendizaje del alumnado. También se ha comentado, que supone una limitación comparar entre distintas cohortes de estudiantes habiendo habido un profesor nuevo en la asignatura que se ha hecho cargo de uno de los grupos. Igualmente, comparar entre cohortes de estudiantes distintos sin establecer las condiciones de experimentación necesarias previamente, constituye una limitación intrínseca al diseño y desarrollo de esta experiencia de innovación docente.

Sobre la sostenibilidad del trabajo, es posible resaltar que la única innovación que ha supuesto un coste real económico, ha sido aquella referente a la disposición de materiales electrónicos y técnicos para el grupo especial de trabajo en interfaces imaginadas, los cuales han contado con un presupuesto de unos 400 euros para gastar en materiales a utilizar durante la implementación de su interfaz imaginada. El resto de innovaciones no ha supuesto un coste económico más allá del coste de las horas extras empleadas por los docentes y colaboradores del proyecto para la elaboración de materiales y organización de las diversas actividades. Por ello, es posible afirmar que la mayoría de innovaciones introducidas son sostenibles a lo largo de distintos años, o aplicables a otras asignaturas que puedan tener características similares (siempre y cuando haya un equipo de trabajo implicado realmente en el proceso).

\section{AgRADECIMIENTOS}

Los autores agradecen al Vicerrectorado de Docencia de la Universidad de Salamanca la financiación recibida para llevar a cabo los proyectos de innovación docente ID2016/048 e ID2016/231 que han permitido llevar a cabo estas nuevas 
experiencias. Del mismo modo, el autor Juan Cruz-Benito y agradece al Fondo Social Europeo y a la Consejería de Educación de la Junta de Castilla y León (España) la financiación de su contrato pre-doctoral. Por su parte, la autora Felicidad García-Sánchez agradece, del mismo modo, a la Universidad de Salamanca y al Banco Santander la financiación de su contrato pre-doctoral como investigador en formación.

\section{REFERENCIAS}

Abascal, J., Aedo, I., Cañas, J. J., Gea, M., Gil, A. B., Lorés, J., . . . Vélez, M. (2001). La Interacción PersonaOrdenador: AIPO (Asociación Interacción Persona Ordenador).

Golbeck, J. (2017). 'Back off, man. I'm a scientist.': using fiction to teach beginners HCI. interactions, 24(2), 7073. doi:10.1145/3029599

Hewett, T. T., Baecker, R., Card, S., Carey, T., Gasen, J., Mantei, M., .. . Verplank, W. (1992). ACM SIGCHI curricula for human-computer interaction: ACM.

Jordan, P., Mubin, O., \& Silva, P. A. (2016). A conceptual research agenda and quantification framework for the relationship between science-fiction media and humancomputer interaction. Paper presented at the International Conference on Human-Computer Interaction.

Marcus, A. (2013). The history of the future: sci-fi movies and HCI. interactions, 20(4), 64-67.

Marcus, A. (2014). Cross-cultural user-experience design for work, home, play, and on the way. Paper presented at the SIGGRAPH Asia 2014 Courses.
Marcus, A. (2015). HCI Sci-Fi at the Movies and on TV HCI and User-Experience Design: Fast-Forward to the Past, Present, and Future (pp. 205-210). London: Springer London.

Michavila, F., Martínez, J. M., Martín-González, M., GarcíaPeñalvo, F. J., \& Cruz-Benito, J. (2016). Barómetro de Empleabilidad y Empleo de los Universitarios en España, 2015 (Primer informe de resultados).

Norman, D. (1988). The psychology of everyday things: Basic books.

Norman, D. (2013). The design of everyday things: Revised and expanded edition: Basic Books (AZ).

Norman, D., \& Draper, S. (1986). User Centered System Design: New perspectives on human-computer interaction.

Schmitz, M., Endres, C., \& Butz, A. (2008). A survey of human-computer interaction design in science fiction movies. Paper presented at the Proceedings of the 2nd international conference on INtelligent TEchnologies for interactive enterTAINment.

Sterling, B. (2013). Interview with Sci-Fi Author Bruce Sterling: Alien-Computer Interfaces. Retrieved from http://uxpamagazine.org/interview-with-bruce-sterling/

Underkoffler, J. S., Parent, K. T., \& Kramer, K. H. (2009). System and method for gesture based control system: Google Patents. 